Conclusion The apparent causal effect of maternal net weight 'gain' on birthweight (and hence macrosomia) is difficult to identify because the total maternal weight gain observed includes that of the offspring. A tautological association is therefore observed even when maternal weight has no causal effect on birthweight. Existing evidence regarding the 'effect' of GWG on birthweight should therefore be viewed with caution and should not be used to inform guidelines on 'ideal' gains in weight.

\section{RF33 EVALUATION OF PUBLIC HEALTH INTERVENTIONS USING A COMPLEX SYSTEMS LENS: A CRITICAL REVIEW}

${ }^{1}$ E McGill, ${ }^{1} \mathrm{~V}$ Er*, ${ }^{2} \mathrm{~T}$ Penney, ${ }^{1} \mathrm{M}$ Egan. ${ }^{1}$ Public Health and Policy, LSHTM, London, UK; ${ }^{2}$ UKCRC Centre for Diet and Activity Research (CEDAR), University of Cambridge, Cambridge, UK

\subsection{6/jech-2019-SSMabstracts. 148}

Background There has been a growth in interest in applying systems thinking to public health research: including greater consideration of the complex and changing nature of realworld environments within which public health interventions take place. In this paper we present the results from a critical review that asked the question: how can a systems approach be applied in the context of public health evaluation?

Methods A critical review of the literature was conducted to identify contrasting examples of systems approaches for indepth comparison and analysis. To inform our protocol and identify relevant studies we held consultations with international researchers with relevant expertise $(n=32)$. We tracked citations from previous reviews and searched Scopus, Medline and Web of Science from 01/01/14 to 06/08/17. We used search terms relating to systems and complexity, evaluation, public health and its social determinants. Our inclusion criteria were as follows: studies must (i) self-identify as taking a systems or complexity-informed approach; and (ii) evaluate one or more interventions or changes in a public health relevant field. Study selection, appraisal, data extraction and analysis were conducted independently by at least two reviewers with regular meetings to discuss contrasting viewpoints.

Results Forty-four studies were finally included. Public health topics varied: the most common concerned obesity, transport, education, and tobacco. Evaluations were classified by the systems approach taken; in total 6 approaches were identified: qualitative research $(n=13)$, concept mapping $(n=3)$, network analysis $(n=4)$, system dynamics modelling $(n=15)$, agentbased modelling $(\mathrm{n}=8)$, and 'systems friendly' approaches $(n=5)$. These different approaches were used to address different research questions but there was also cross-over between methodological approach and purpose. Some studies lacked clear empirical conclusions for informing future practice.

Discussion Though sometimes portrayed as a novel development in public health research, there are already numerous examples of different public health 'systems evaluations'. There is no single or dominant 'systems approach' to public health evaluation. Nor is there a consistent pattern whereby different approaches address research questions specific to that approach. Rather than advocate for a single approach to systems evaluation, we believe continued innovation in this field is most helpful at this time. To improve utility, some systems evaluations would benefit from improvements in the reporting of empirical findings.

\section{RF34 SOCIOECONOMIC DIFFERENCES IN CARDIOVASCULAR DISEASE RISK FACTOR PREVALENCE IN PEOPLE WITH TYPE 2 DIABETES IN SCOTLAND: A CROSS-SECTIONAL STUDY}

${ }^{1} \mathrm{E}$ Whittaker*, ${ }^{2} \mathrm{SH}$ Wild. 'Edinburgh Medical School, University of Edinburgh, Edinburgh, UK; ${ }^{2}$ Usher Institute of Population Health Sciences and Informatics, University of Edinburgh, Edinburgh, UK

\subsection{6/jech-2019-SSMabstracts. 149}

Background Health inequalities exist in outcomes of diabetes in different socioeconomic groups and these are particularly marked for cardiovascular disease. This study explores the association between socioeconomic status (SES) and prevalence of cardiovascular risk factors (smoking, body mass index (BMI), glycated haemoglobin $\left(\mathrm{HbA}_{1 \mathrm{C}}\right)$, blood pressure and cholesterol) in people with type 2 diabetes in contemporary Scottish data.

Methods We performed a cross-sectional study of people with type 2 diabetes in Scotland who were alive on 30/6/16 identified from the population-based diabetes register. SES was defined using quintiles of the area-based Scottish Index of Multiple Deprivation (SIMD). Cardiovascular risk factors were defined as: current smoker, obesity (BMI $\geq 30 \mathrm{~kg} / \mathrm{m} 2$ ), $\mathrm{HbA}_{1 \mathrm{C}} \geq 58 \mathrm{mmol} / \mathrm{mol}$, systolic blood pressure $\geq 140$ $\mathrm{mmHg}$, and cholesterol $\geq 5 \mathrm{mmol} / \mathrm{l}$. Logistic regression models adjusted for age, sex, health board, history of cardiovascular disease, ethnicity and duration of diabetes were used to identify odds ratios (OR) (95\% confidence intervals) for the most compared to the least deprived SIMD quintile for each risk factor.

Results There were 264,664 people with type 2 diabetes in the study population: mean (SD) age was 66.7 (12.8) years, $56.1 \%$ were male, $23.6 \%$ were in the most deprived quintile and $15.1 \%$ in the least deprived quintile. Less than $5 \%$ had missing data on key variables.

Crude prevalence of risk factors in the most/least deprived quintiles were $24.4 / 8.8 \%$ for smoking, $61.9 / 49.4 \%$ for obesity, $43.7 / 39.7 \%$ for above-target $\mathrm{HbA}_{1 \mathrm{C}}, 30.5 / 31.3 \%$ for above-target systolic blood pressure and $24.4 / 24.5 \%$ for above-target cholesterol.

Adjusted prevalence of current smoking (OR 3.25 (95\% CI 3.09-3.42)), obesity (OR 1.59 (1.54-1.65)) and above-target $\mathrm{HbA}_{1 \mathrm{C}}$ (OR 1.13 (1.09-.17)) were higher in the most compared to the least deprived quintile. Adjusted prevalence of above-target systolic blood pressure was similar (OR 1.00 (0.97-1.04)), and of above-target cholesterol was lower in the most compared to the least deprived quintile (OR 0.85 (0.82$0.88)$ ). Inequalities in current smoking were larger in females (OR 3.66 (3.37-3.96)) compared to males (OR 3.00 (2.813.19)). No other risk factors showed interactions between sex and SES.

Conclusion Socioeconomic deprivation is associated with significantly higher prevalence of smoking, obesity, and above-target $\mathrm{HbA}_{1 \mathrm{C}}$ among people with type 2 diabetes in Scotland. Inequalities in smoking status by SES among people with type 2 diabetes appear to have widened over time. Effective approaches to reducing these inequalities at both population 
and individual levels are required as well as reducing prevalence of risk factors across the whole population.

\section{RF35 SUPPORT THAT COMES FROM CULTURE: A RASCH ANALYSIS OF THE SOCIAL SUPPORT SCALE (SSS) IN AN ABORIGINAL POPULATION}

${ }^{1}$ PHR Santiago*, ${ }^{2} \mathrm{~L}$ Smithers, ${ }^{3} \mathrm{R}$ Roberts, 'L Jamieson. 'Adelaide Dental School, The University of Adelaide, Adelaide, Australia; ${ }^{2}$ School of Public Health, The University of Adelaide, Adelaide, Australia; ${ }^{3}$ School of Psychology, The University of Adelaide, Adelaide, Australia

\subsection{6/jech-2019-SSMabstracts. 150}

Background Due to a history of colonization, Aboriginal and Torres Strait Islander became one of the most disadvantaged groups in Australia. The decades of assimilation policies, which culminated in the removal of Aboriginal children from their parents, disarranged the social support derived from their kinship system. In non-Indigenous populations, social support has been reported as a protective factor against stress (i.e. buffering hypothesis) and coronary heart disease; providing also better outcomes for breast cancer and type- 2 diabetes. There are no psychological instruments validated to measure social support in Aboriginal Australians. The aim of the current study was to evaluate the validity and reliability of the Social Support Scale in an Aboriginal population.

Methods The Social Support Scale (SSS) is a 4-item measure developed to evaluate the emotional, appraisal, instrumental and informational domains of social support. Data was collected from to two different samples: participants of the (1) Teeth Talk Study $(n=317)$, an oral-health randomized controlled trial (RCT) conducted among Aboriginal adults; and (2) the Baby Teeth Talk Study $(n=367)$, an RCT conducted among pregnant Aboriginal women. The psychometric properties of the SSS were evaluated with Graphical Loglinear Rasch Models (GLLRM), which consist of recent methodological advances that extend the Rasch Model to incorporate local dependence (LD) and differential item functioning (DIF).

Results Overall fit to a GLLRM was found $\left(X^{2}(96)_{\text {sample } 1}=52.7\right.$, $\left.\mathrm{p}=0.057 ; \mathrm{X}^{2}(25)_{\text {sample } 2}=22.2, \mathrm{p}=0.625\right)$ after the inclusion of LD between items 3 and $4\left(g_{a v g 1}=0.50 ; g_{a v g 2}=0.66\right)$. Item 2 displayed DIF by employment status $\left(\mathrm{X}^{2}(4)=12.60, \mathrm{p}=0.013\right.$, $g=0.21)$ in Sample 1. There was no DIF by sex or educational attainment. Unidimensionality was confirmed in both samples $\left(\mathrm{g}_{\mathrm{obs} 1}=0.80 ; \quad \mathrm{g}_{\exp 1}=0.78, \mathrm{p}=0.654 ; \mathrm{g}_{\mathrm{obs} 2}=0.75, \mathrm{~g}_{\exp 2}=0.77\right.$, $\mathrm{p}=0.163)$. The SSS displayed good reliability $\left(\mathrm{R}_{\text {sample } 1}=0.82\right.$, $\left.\mathrm{R}_{\text {sample } 2}=0.84\right)$ and probability of person separation $\left(\mathrm{P}_{\text {sample } 1}=0.77\right.$, $\left.\mathrm{P}_{\text {sample } 2}=0.78\right)$. Targeting was poor $\quad\left(\mathrm{TTI}_{\text {sample } 1}=0.28\right.$; $\mathrm{TTI}_{\text {sample2 }}=0.49$ ).

Conclusion The same unidimensional structure was found in both samples, providing confidence in the robustness of results. Item 2 ('There are people in my life who appreciate what I do') displayed DIF by employment status, indicating that participants who were employed were more likely to feel appreciated than those unemployed given the same level of social support. The current study consisted of the first validation of a Western-developed psychological instrument to measure social support in Aboriginal Australians. The results show that the SSS is a culturally-valid and reliable instrument that can be applied in future health research among Aboriginal and Torres Strait Islanders.

\section{RF36 COMPARING MULTIPLE FORMS OF DISCRIMINATION AND POSTPARTUM DEPRESSION AMONG PALESTINIAN-ARAB MINORITY WOMEN, IMMIGRANT AND NON-IMMIGRANT JEWISH WOMEN IN ISRAEL}

${ }^{1} \mathrm{~N}$ Daoud*, ${ }^{1} \mathrm{R}$ Sergienko, ${ }^{2} \mathrm{M}$ Geo. 'Department of Public Health, Ben-Gurion University of the Negev, Beer-Sheva, Israel; ${ }^{2}$ Critical Care Services, Critical Care Services, Toronto, Canada

\subsection{6/jech-2019-SSMabstracts. 151}

Background Drawing on the intersectionality theory, emerging research shows that discrimination is a multidimensional risk factor for health. Yet, associations between multiple forms of discrimination (MFD) and postpartum depression (PPD) have not been explored. This study compares relationships between MFD and PPD among Palestinian-Arab indigenous minority, Jewish immigrant, and Jewish non-immigrant mothers citizens of Israel.

Methods We used data from a stratified sample of 1,128 postpartum mothers who were interviewed during visiting maternal and child health clinics in 2014-15. We conducted multivariable logistic regression analysis and generalized estimation equation for PPD (Edinburgh Postnatal Depression Scale with cutoff $\geq 10$ ) and compared associations with two measure of MFD among the study groups, while considering age, socioeconomic status, anti-depressant use, and single forms of discrimination in different models. The two MFD measures included: cumulative MFD (additive experiences of $0,1,2$ or $\geq 3$ forms of discrimination based on ethnicity, skin color, religiosity level, gender, age and socioeconomic status), and composite MFD (12 categories resulting from an interactions terms between cumulative $0,1,2$ and $3 \geq$ MFD and women's study groups. The reference category was non-immigrant Jewish mothers who reported no MFD.

Results Palestinian-Arab mothers reported highest MFD, followed by Jewish immigrant mothers and non-immigrant Jews $(\geq 3 \mathrm{MFD}=29.2 \%, 24.1 \%$ and $17.8 \%$, respectively). Composite MFD had stronger dose response associations with PPD among Palestinian-Arab women, followed by immigrant Jews and non-immigrant Jewish women. Compared to non-immigrant Jewish women with no MFD, Palestinian-Arab women reporting $\geq 3 \mathrm{MFD}, 2 \mathrm{MFD}$ and one MFD were more likely to experience PPD. Adjusted odds ratio and 95\% confidence intervals (AOR, 95\%CI) were 12.68 (5.29-30.40), 10.08 (3.73-27.20), and 3.98 (1.23-12.86), respectively, among Palestinian-Arab women; 4.44 (1.45-13.61), 5.76 (1.84-17.97), 2.32 (0.59-9.12), respectively, among immigrant Jewish mothers; and 4.68 (1.87-11.71), $3.74(1.32-10.63)$ and 2.70 (1.06-6.87) among non-immigrant Jews mothers. Cumulative or additive MFD showed a strong dose response association with PPD among non-immigrant Jews and Palestinian-Arab women who reported $\geq 3 \mathrm{MFD}$, but not among immigrant Jewish women.

Conclusion The study result sheds light on the importance of studying the facets of MFD in intersection with social 